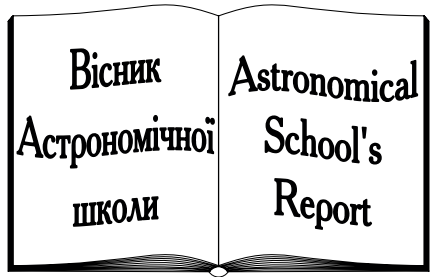

ISSN 2411-6602 (Online)

ISSN 1607-2855 (Print)

Volume $11 \bullet$ Issue $2 \bullet 2015 \quad$ P. $135-138$

UDC 523.4-327

\title{
The dependence of the YORP effect on an asteroid shape
}

\author{
U.V. Pyrohova ${ }^{1 *}$, O.A. Golubov ${ }^{1,2}$ \\ ${ }^{1}$ Institute of Astronomy of Kharkiv National University \\ ${ }^{2}$ V.N. Karazin Kharkiv National University
}

In this paper we deal with the problem of analytic calculation of the YORP effect of an asteroid in terms of its shape. The calculations are made for spheroidal asteroids, whose shape slightly differs from a triaxial ellipsoid. We analytically describe a real asteroid as a triaxial ellipsoid perturbed with spherical harmonics. The results can be applied to predict the YORP torque for asteroids with specified shape, as well as to understand contribution of roughness on different scales to the YORP effect.

ЗАЛЕЖНІСТЬ YОRР ЕФЕКТУ ВІД ФОРМИ АСТЕРОЇДА, Пирогова У.В., Голубов О. - У иій статті ми розглядаємо проблему аналітичного розрахунку YORP ефекту астероїда виходячи з його форми. Розрахунки виконані для сферичних астероїдів, чиї форми трохи відрізняються від тривісного еліпсоїда. Реальний астероїд аналітично описаний як тривісний еліпсоїд, збурений сферичними гармоніками. Отримані результати можуть бути застосовані для прогнозування YORP моменту астероїдів з відомою формою, а також для оцінки внеску в YORP шорсткостей різних масштабів.

ЗАВИСИМОСТЬ YОRР ЭФФЕКТА ОТ ФОРМЫ АСТЕРОИДА, Пирогова У.В., Голубов О. - В этой статье мь рассматриваем проблему аналитического расчета YORP эффекта астероида исходя из его формь. Расчеть выполнены для сферических астероидов, чьи формы немного отличаются от трехосного эллипсоида. Реальный астероид аналитически описан как трехосный эллипсоид, возмущенный сферическими гармониками. Полученные результаты могут быть применены для прогнозирования YORP момента астероидов с известной формой, а также для оценки вклада в YORP шероховатостей разных масштабов.

Ключевые слова: астероиды; YORP эффект.

Key words: asteroids; YORP effect.

\section{INTRODUCTION}

In the 20th century collisions and gravitational interactions were considered the governing mechanisms of asteroid motion. This suggestion is called the classical theory of evolution of celestial bodies [2]. Using the classical theory, the evolution history of the main belt and near-Earth asteroid population was reconstructed. Unfortunately, there are lots of discrepancies of the classical theory and observational data.

For example, from the classical theory it follows that due to collisions asteroids should have Maxwellian distribution over their angular momenta. But this suggestion holds true only for big asteroids with diameters $D>40 \mathrm{~km}$ [1], while for smaller asteroids distribution of angular momenta is more regularized. It is not possible to describe this observational data just using the paradigm of collisions.

Thus, it is needed to learn more about other process acting on asteroids [2].

As Paddack showed in his work [3], emission of light from the asteroid surface can change angular momentum of a body. It could be significant if an asteroid shape is asymmetric like a propeller or a windmill. Paddack recognized that when the sunlight falls on the surface and is re-emitted by it, it creates some torque on each part of the surface. If the surface is asymmetrical with respect to its main axis of rotation like a propeller or a windmill, then the total torque can be non-zero. Thus, the spin of the asteroid could be changed due to the asteroid asymmetry.

Paddack named this torque Windmill effect.

The amount of the torque that is given by light to the massive asteroid is tiny due to the small value of photon impulse. But this torque acts on the asteroid perpetually, and its effect piles up for millions of years. The less is the asteroid mass the faster it rotates due to Windmill effect [6]. Therefore, on the scale of million years the Windmill effect dominates over collisions only for small asteroids, with typical diameters $D<10 \mathrm{~km}[1]$.

Nowadays, the described Windmill effect is called the YORP effect, or Yarkousky-O'Keefe-RadzievskiiPaddack effect [4], the name composed by Rubincam [6] from the first letters of names of the people who pioneered the study of non-gravitational forces acting on celestial bodies.

* Pyrohova Ulyana Vladimirovna; $\$ ulyana487@gmail.com

Astronomical School's Report, 2015, Vol. 11, Issue 2 
Statler was the first to notice that the YORP effect is sensitive to small scale roughness of an asteroid [5]. He showed that the YORP torque experienced by an asteroid significantly changes when a crater or a bounder appears on its surface, even if the crater or the boulder is an order of magnitude less than asteroid size. The main result of Statler's work was the demonstration of the importance of small scale structures. Still, no sophisticated study of relative importance of structures at all scales has been performed. And in this work we are aiming to do the next step in this direction.

\section{CALCULATION OF THE YORP TORQUE}

To calculate YORP from an asteroid shape let us calculate the YORP torque of each asteroid irregularity, and summarize them. Let us make a mathematical model describing the shape of a real asteroid.

\subsection{Mathematical description of a real asteroid}

As most asteroids have substantial elongations, from afar it appears like a triaxial ellipsoid. When we look at the asteroid closer, it could be treated as a distorted triaxial ellipsoid. According to the notion that every shape could be defined as a certain set of spherical harmonics, let us consider each asteroid as a triaxial ellipsoid perturbed with a certain set of spherical harmonics.

Let us imagine a triaxial ellipsoid as an elongated sphere. Then in reference frame of an asteroid mass center, the radius-vector of some point on the asteroid has the following length,

$$
\overrightarrow{\mathbf{r}}=\rho(\alpha \beta \sin \varphi \sin \vartheta, \quad \beta \cos \varphi \sin \vartheta, \quad \cos \vartheta),
$$

with $\alpha$ and $\beta$ being coefficients that show the asteroid's elongation on two main axes, and $\varphi$ and $\vartheta$ being two free parameters. Let the axis $z$ be the rotation axis of the asteroid, i.e. the shortest one. And let the axis $x$ be no longer than the axis $y$, i.e. $\alpha \beta \geqslant \beta$.

Let us perturb the surface, $\rho=\rho_{0}+\widetilde{\rho}$, where $\widetilde{\rho}$ describes a slight distortion $\rho_{0} \gg \widetilde{\rho}$ of the elongated sphere with a set of spherical harmonics.

Spherical harmonics that do not change the the ellipsoid shape should be excluded from the set. The spherical harmonics $Y_{00}$ only changes $\rho_{0}$; harmonics $Y_{m 1}$ shift the asteroid's center of mass; harmonics $Y_{m 2}$ elongate the asteroid into a thiaxial ellipsoid, but we include the elongation into the parameters $\alpha$ and $\beta$. Thus, we only include harmonics with $l \geqslant 3$ into the decomposition. Then we have

$$
\rho=\rho_{0}+\widetilde{\rho}=\rho_{0}\left(1+\frac{1}{\rho_{0}} \sum_{l=3}^{\infty} \sum_{m=0}^{m=l} a_{m l} Y_{m l}\right),
$$

$a_{m l}$ are coefficients specific for each body; $Y_{m l}$ are spherical functions.

Physical sense of indices $l$ and $m$ is the following:the difference $(l-m)$ is equal to the number of nodes in the latitudinal direction along the asteroid, while $m$ is equal to the number of nodes in the longitudinal direction.

\subsection{The method of calculations}

As most asteroids are very dark, we assume the asteroid to be a black body.

It is known that in depth of regolith the thermal conductivity of the order of $10^{-3} \frac{\mathrm{W}}{\mathrm{m}^{2} K^{2}}$. The effect of nonzero thermal conductivity in boulders with radius less or of the order of one meter produces a significant contribution to the YORP torque. But thermal conductivity is non-essential for surface irregularities on bigger scales. Therefore, we assume the conductivity of the regolith equal to zero.

We assume the perturbation of the shape to be slight, so that the asteroid stays convex.

Assume asteroid obliquity is equal to zero, i.e. the its rotation axis is perpendicular to its equatorial plane. We also assume that the asteroid does not precess.

Let $\overrightarrow{\mathbf{e}}_{z}$ be the ort of the rotation axis of the asteroid, and $\overrightarrow{\boldsymbol{\tau}}$ the total YORP toque averaged over the rotation period. As we assume obliquity to be equal to zero, vector of the YORP torque is coaxial with the rotation axis $\overrightarrow{\boldsymbol{\tau}} \| \overrightarrow{\mathbf{e}}_{z}$ and $\overrightarrow{\boldsymbol{\tau}}=\left(0,0, \tau_{z}\right)$. Let us now compute the YORP torque $\tau_{z}$ of a slightly curved triaxial ellipsoid. The total torque averaged over the rotation period is

$$
\tau_{z}=\int d \tau_{z}=\int(\overrightarrow{\mathbf{r}} \times d \overrightarrow{\mathbf{F}}) \overrightarrow{\mathbf{e}}_{z}
$$

where $d \overrightarrow{\mathbf{F}}$ is the force acting on an element of the asteroid surface with the radius-vector $\overrightarrow{\mathbf{r}}$.

Light scattered according to Lambert's law pushes the surface in perpendicular direction. Correspondingly, the force vector $d \overrightarrow{\mathbf{F}}$ is aimed at the surface, i.e. $d \overrightarrow{\mathbf{F}}$ has the direction opposite to the surface normal $d \overrightarrow{\mathbf{S}}$. $\overrightarrow{\mathbf{F}}=-p d \overrightarrow{\mathbf{S}}$ is pressure pressure force acting on a small element of the surface $d \overrightarrow{\mathbf{S}}=\frac{\partial \overrightarrow{\mathbf{r}}}{\partial \varphi} \times \frac{\partial \overrightarrow{\mathbf{r}}}{\partial \vartheta}$, with $p$ being the pressure. For $p$ we use the following expression,

$$
p_{z}=p_{0} \cos \psi,
$$

where $\psi=\frac{\pi}{2}-\arccos \left(\frac{d \overrightarrow{\mathbf{S}}}{d S} \overrightarrow{\mathbf{e}_{\mathbf{z}}}\right)$ is the angle between the normal of the surface element $d \overrightarrow{\mathbf{S}}$ and the equatorial plane. 
We summarize the torques created by all surface elements and get the total torque experienced by the asteroid. Accordingly to our way of description of an asteroid shape the asteroid total YORP torque is the sum of YORP torques $\tau_{z l m}$ of spherical harmonics that make asteroid slightly perturbed,

$$
\tau_{z}=\int d \tau_{z}=\oint(d \overrightarrow{\mathbf{S}} \times \overrightarrow{\mathbf{r}}) \overrightarrow{\mathbf{e}_{\mathbf{z}}} p_{z}=\sum_{l=3}^{\infty} \sum_{m=0}^{m=l} a_{l m} \tau_{z l m}
$$

Here $a_{l m}$ are coefficients that are specific for each asteroid. This equation expresses the YORP torque experienced by the asteroid in terms of its shape. The coefficients $\tau_{z l m}$ can be expressed analytically, although the expressions are lengthy. It is more instructive to discuss $\tau_{z l m}$ qualitatively and to study them numerically.

\section{CONTRIBUTION OF DIFFERENT HARMONICS TO THE YORP TORQUE}

Let us consider the torque $\tau_{z m l}$ of an ellipsoid perturbed by spherical function $Y_{m l}$.

Remember that $x, y, z$ are coordinates in the reference frame of the asteroid mass center. It is evident that the sign of $z$-component of the torque changes when the asteroid is inverted with respect to any plane containing $z$ axis. But if this plane is the plane of mirror symmetry of the asteroid, then the inversion transforms the asteroid to itself, and the torque is not changed. To satisfy these two conditions simultaneously, the torque should be equal to zero.

Thus all spherical harmonics with cosine vanish, as they are symmetric with respect to the plane $x z$, and only the harmonics with sine survive. Among them, harmonics with odd $m$ vanish, as they are symmetric with respect to the plane $y z$. Ultimately, harmonics with odd $l$ and even $l$ vanish, as they transform into themselves after mirror reflections in planes $x z$ and $x y$. (Reflection in the plane $x y$ leaves the YORP torque intacked.)

Therefore, only spherical harmonics $Y_{m l}$ with even indexes of $m$ and $l$ contribute non-zero torque $\tau_{z m l}$. In Fig. 1 are shown first five harmonics with non-zero YORP. In Fig.2 is shown the dependence of $\tau_{z m l}$ for these harmonics on elongation of the asteroid. As $l$ and $m$ increase the asymmetric property of perturbed with $Y_{m l}$ ellipsoid decreases. Correspondingly, its YORP torque $\tau_{z m l}$ also deceases. The dependence of YORP effect on elongation $\alpha$ is much stronger than on $\beta$.
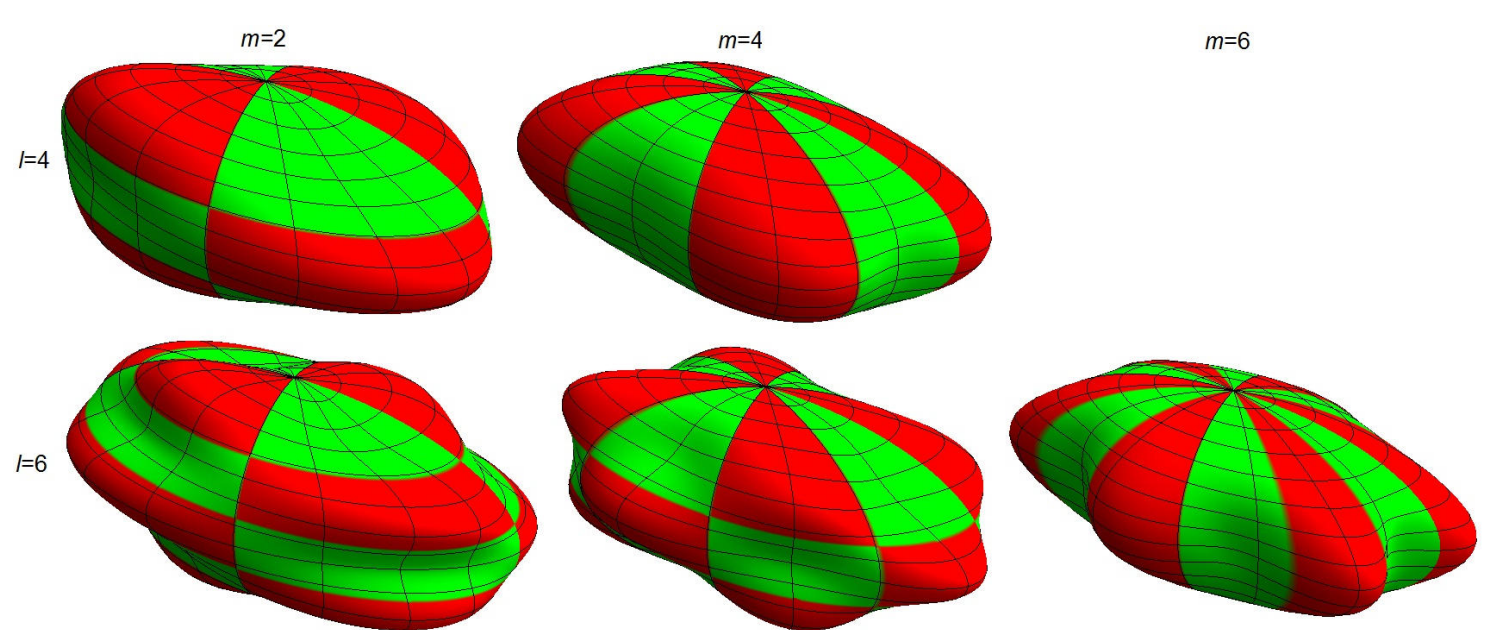

Fig. 1. Even harmonics $Y_{m l}$ with $(l, m)=(4,2),(4,4),(6,2),(6,4),(6,6)$, contributing non-zero YORP torque $\tau_{z m l} \neq 0$

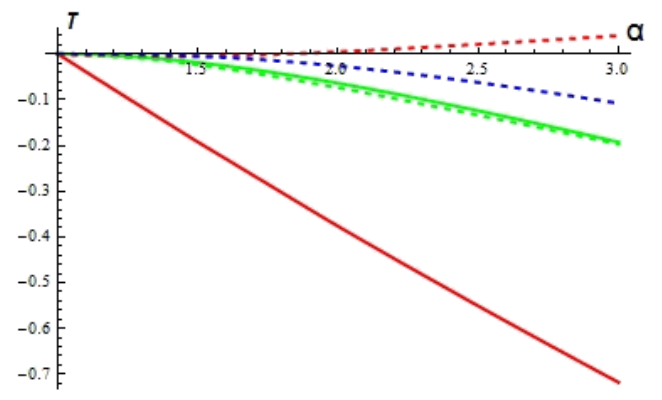

$\beta=1$

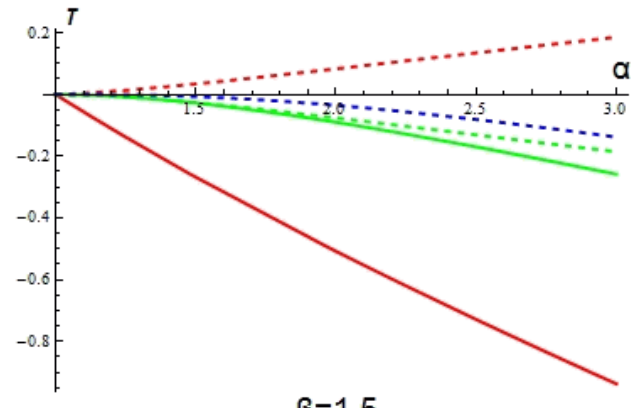

$\beta=1.5$

Fig. 2. The dependence of the YORP effect on elongation $\alpha$ for different harmonics. The left panel corresponds to $\beta=1$, the right panel to $\beta=1.5$. Cases of $l=4$ are shown with solid curves, and $l=4$ with dashed curves. Harmonics with $m=2$ are colored in red, $m=4-$ green, and $m=6-$ blue. 


\section{CONCLUSION}

In this paper was investigated the YORP effect acting on an asteroid, which shape is represented as a series of spherical harmonics. The resultant moment of forces of light pressure was also expressed in a series of individual spherical harmonics.

It was assumed that the average pressure of reinsolated light acting on an asteroid surface element is proportional to the cosine of the latitude. And the expression of the torque generated by the individual harmonics was obtained in quadratures.

The results can be used to calculate the YORP effect acting on asteroids with specified shape. But more essential application of the obtained decomposition of the torque in spherical functions is analyzing of the contribution of different scaled surface roughnesses in the YORP effect. It is also needed for evaluation of an error, which could appear in a value of YORP torque when the higher harmonics are discarded in simplified shape models that are derived from observational data.

1. Pravec P., Harris A. W., Michalowski T., 2002, Asteroid rotations.

2. Bottke W.F., Vokrouhlick D., Rubincam D.P., Nesvorn M.D. The Yarkovsky and Yorp effects: Implications for asteroid dynamics // Annu. Rev. Earth Planet. Sci. - 2006. - 34. - P.157-191.

3. Paddack S.J. Rotational bursting of small celestial bodies: Effects of radiation pressure // J. Geophys. Res. - 1969. - 74. - P.4379-4381.

4. Vokrouhlicky D., Bottke W.F., Chesley S.R., Scheeres D.J., Statler T.S. // 2015, arXiv:1502.01249v1.

5. Statler T.S. // Icarus. - 2009. - 202. - P.502.

6. Rubincam D.P. Radiative spin-up and spin-down of small asteroids // Icarus. $\mathbf{2} 2000$. $\mathbf{1 4 8}$. - P.2.

Received 9.11.2015

Accepted 10.12.2015 